\title{
Ueber die Entwickelung der Nerven aus Plasmazellen beim Frosche.
}

\author{
Von
}

Dr. Korybutt-Daszkiewicz aus Warschau.

\author{
Hierzu Tafel I.
}

Im Verlauf einer Untersuchung ther die Entwickelung der peripherischen Nerven kam ich auf den Gedanken, die bei Frihlings-Fröschen eintretende Neubildung der Gewebe auch in Bezug auf die Nerven zu untersuchen.

Bereits bei den ersten, Frihjahrs-Fröschen entnommenen Objecten, bekam ich Gebilde zu Gesicht, welche mir bisher nur als embryonale Nervenfasern bekannt, und die, je weiter in den Sommer hinein, in allen Stufen der Entwickelung bis zur histologischen Reife anzutreffen waren. Einmal auf das Vorkommen dieser Neubildung von Nervenfasern aufmerksam gemacht, galt es nun festzustellen, welchen histologischen Elementen dieselbe ihre Entstehung verdanken. Nach zahlreichen Untersuchungen ist es mir gelungen, in den, zwischen den uberwinternden Fasern vorkommenden Bildungs- oder Plasmazellen (W aldeyer) diejenigen Gewebselemente zu erkennen, welche der Neubildung der peripherischen Nervenfasern zu Grunde liegen. Ehé ich zur Schilderung der einschlägigen Beobachtungen ubergehe, sei es mir gestattet, einige Data tiber die, fur den vorliegenden Gegenstand so wiehtigen Plasmazellen, vorauszuschicken. 
Diese Gebilde wurden zuerst von v. Recklinghausen ${ }^{1}$ ) im Omentum und der Pleura junger Kaninchen gesehen, und als grosse, eigenthumliche Bindegewebskörperchen beschrieben. Bald darauf fand $K u \mathfrak{h n} \mathrm{e}^{2}$ ) im intermuskulären Bindegewebe des Frosches Zellen, welche er als grobkörnig, im durchfallenden Lichte tribe, im auffallenden weisslich aussehend, bezeichnete und ebenfalls den Bindegewebselementen zuzählte; ferner wies dieser Beobachter nach, dass die körnige Beschaffenheit der Zellen nicht von Fettmolectilen herrihhre, und dass ibrem Protoplasma die Contractilität abginge. In der Folge hat $\mathrm{Cohnheim}^{3}$ ) in der Zunge des Frosches ähnliche Gebilde gesehen, deren mannigfaltige Formen ihm auffielen, und die er, ohne sie mit den $\mathrm{K} u \mathrm{hn}$ e'schen Zellen zu identificiren, als besondere Bindegewebselemente betrachtete. Rollett ${ }^{4}$ ) erwähnt dieser Zellen im Unterschenkel des Frosches, und schrieb ihnen eine tiberwiegend längliche Form und das Bestreben zu, mit ihren spitzen Enden unmittelbar an einander zu stossen. v. Biesiadecki $\mathrm{i}^{5}$ fand sie im Unterhautzellgewebe und zwar vorherrsehend im Verlauf der Blutgefässe. Nach E. Klein ${ }^{6}$ ) kommen tiberall in den bindegewebigen Organen, wie auch in serösen Häuten, rundliche, granulirte Protoplasmaklumpen mit einem oder mehreren Kernen vor, welche, $z w$ ischen den ge streckt verlaufenden Bindegewebsbundeln der serösen Häute, eine mehr spindelförmige Form annehmen. Auch in den Scheiden der dicken Froschnerven sind sie von diesem Forscher angetroffen worden ${ }^{7}$ ).

Die Mannigfaltigkeit der Form und ihres Vorkommens könnte dem Gedanken Raum geben, als seien die, von den Autoren in den verschiedenen Organen aufgefundenen Zellen heterogene Gebilde. Daher musste es als ein Fortschritt betrachtet werden, als Wal-

1) v. Re ck ling h a u s e n. Ueber Eiter- und Bindegewebskörperchen. Virchow's Archiv 28. Bd.

2) Kühne. Ueber das Protoplasma. Leipzig 1864.

3) Cohnheim. Ueber das Verhalten der fixen Bindegewebszellen. Virch. Arch. 45. Bd.

4) Rollett. Stricker's Hdb. d. Gwblehre. I. Bd. S. 40.

5) v. Biesia decki ibidem. S. 583.

6) E. Klein ibidem S. 621 .

7) Derselbe. Handbook for the phys. Labor. London S. 37. 
Ueber die Entwickelung der Nerven aus Plasmazellen beim Frosche. 3

deyer ${ }^{1}$ ) in allen jenen Zellen dieselben Gewebselemente erkannte, ihr Vorkommen in fast allen Organen nachwies, und dieselben als Bildungszellen des Bindegewebes oder Plasmazellen in die Histologie einfuhrte. Wichtig fur die uns beschäftigende Frage ist der Umstand, dass Waldeyer unter anderem die von Sigmund Mayer ${ }^{2}$ ) als Bildungszellen des Frosch-Sympathicus beschriebenen Gebilde, ebenfalls für Plasmazellen erklärte. Bekanntlich hat Sig. Mayer im Sympathicus und der Nebenniere des Frosches die sogenannten Zellennester beschrieben, welche in letzter Instanz von den rothen Blutkörperchen abstammen und nach der Vermuthung dieses Autors das Material fur Neubildung von Nervenzellen und Fasern liefern sollen; auch sei das Auftreten dieser Zellen, ceteris paribus, von der Jahreszeit und vom Alter des Thieres abhängig. - Abgesehen von den vereinzelten Berichtigungen, welche die Angaben Wald eyer's erfahren haben, können dieselben als Grundlage unserer heutigen Kenntniss dieser Gebilde betrachtet werden.

Der neueste Bearbeiter dieses Gegenstandes, $\mathrm{E} \mathrm{hrli} \mathrm{ch}^{\mathrm{B}}$ ), glaubt, indem er in dem Dahliafarbstoff ein Mittel gefunden, welches die Plasmazellen lebhaft färbt, dieselben dadurch von den anderen Zellen unterscheiden zu können. Nachdem er durch dieses Verfahren die Anwesenheit der Plasmazellen in fast allen Organen mit Ausnahme der Nebenniere und des Hodens (gegen Waldeyer) constatirt, theilt er sie ihrer Form nach in zwei Gruppen, polyedrische und Spindelzellen, wobei er, die letzteren betreffend, hervorhebt, dass die längeren Spindelzellen eine Neigung mit einander zu verschmelzen zeigten, wodurch ausserordentlich lange Gebilde mit mehreren Kernen entständen, die sich meist dem Verlauf der Capillaren anschlössen.

Aus dem Vorhergehenden wird ersichtlich, dass, abgesehen von einzelnen noch zweifelhaften Erscheinungsarten, die Plasmazellen in ihren prägnanten Formen als ein Gewebselement sui generis aufzufassen sind, deren Abstammung allerdings noch unaufgeklärt bleibt, was um so mehr zu bedauern ist, als ja ihre

1) Waldeyer. Ueber Bindegewebszellen. Dieses Archiv XI. Bd.

2) Sigmund Mayer. Beobachtungen und Reflexionen u.s. w. Wiener Acad. Sitz. Berichte. 3. Abth. LXVI Bd. 1872.

3) Ehrlich. Beiträge zur Kenntniss der Anilinfärbung. Dieses Archiv 13. Band. 
Beziehung zur Neubildung der Nerven in einem noch nicht gelösten Widerspruche mit den bisherigen Anschaunngen vieler Embryologen zu stehen scheint.

Ich gehe jetzt zur näheren Begrïndung der Eingangs erwähnten Entstehung der Nerven aus den Plasmazellen iber. Versetzt man einen Frosch, dem lange Zeit (mindestens mehrere Wochen hindurch) die Nahrung entzogen worden, in gttnstigere Verhältnisse, beispielsweise in ein offenes Garten-Aquarium, so zeigen sich schon nach ein paar Wochen tiberall in den verschiedenen Geweben reichliche Plasmazellen. An den Nerven, welche uns zunächst beschäftigen, treten sie zwischen den Fasern und im Verlaufe der Capillaren auf.

Die Zellen sind hier zuerst oval, mit einem bei guter Färbung deutlichem, länglichem Kerne und grobkörnigem, trubem Protoplasma. Nach und nach wachsen diese Zellen, durch die sie unmittelbar begrenzenden Nervenfasern in ihrer seitlichen Ausbreitung beschränkt, in einer zu den Nervenfasern parallelen Richtung, zu mehr oder weniger langen Protoplasmasträngen aus. Dies Wachsthum des Protoplasma findet entweder gleichzeitig an beiden Polen der Zelle statt, oder (wie es Fig. 4 u. 5 zeigt) es erfolgt die Verlängerung nur einseitig, während um den Kern herum ein Theil des Protoplasma sich zu einer Anschwellung sammelt, welche alsdann auf dem verlängerten Theile, gleichsam wie der Kopf auf dem Nagel, aufsitzt; auch diese Ansammlung betheiligt sich in der Folge an der weiteren Entwickelung des Protoplasmastranges. Die nächste Erscheinung ist die Theilung der Kerne und ihr Auseinanderrticken in der Richtung des auswachsenden Protoplasma. Dieser Process wiederholt sich fort und fort, bis die verlängerte Zelle eine neue Plasmazelle berthhrt, und mit ihr zu verschmelzen scheint. Ich sage ausdricklich: „scheint", weil der Act der Verschmelzung selbst sich nicht verfolgen lässt, und andererseits auch durch fortgesetzte Theilung der Kerne das Auswachsen einer einzigen Plasmazelle zu einer Nervenfaser immerhin möglich wäre. Dass jedoch bei der Neubildung von Nerven die Verschmelzung der häufigere Vorgang sein muss, ersieht man aus der grossen Anzahl von Plasmazellen zwischen den Nervenfasern, und dem Mangel an solchen Bildern, welche auf einen Zerfall oder eine anderweitige Verwendung derselben hinwiesen.

Inzwischen nehmen einige der oben beschriebenen protoplas- 
Ueber die Entwickelung der Nerven aus Plasmazellen beim Frosche. 5

matischen Zellenfasern an Dicke bedeutend zu, während andere hingegen diun bleiben. An beiden Arten der Fasern kommt eine zuerst mehr punktförmige, später rein lineare Längsstreifung zum Vorschein, welche auf neben einander liegende Axenfibrillen zu beziehen ist, worauf, neben dem optischen Bilde, auch die Tinctionsfähigkeit für Anilin hinweist (Remak'sche Fasern).

Hand in Hand mit dieser Erscheinung treten einige Kerne aus dem Inneren der Fasern an ihre Oberfläche (was ausnahmsweise schon an den Plasmazellen selbst zu beobachten ist), wobei die Kerne jedoch fortfahren sich zu vermehren, was so lange dauert, bis deren genug vorhanden sind, um für jeden ans dieser Längsstreifung hervorgegangenen Axencylinder als Ausgangspunkt der Schwann'schen Hüllenbildung dienen zu können.

Unmittelbar vor der Bildung des Markes verfliussigt sich die Grundsubstanz dieser Zellenfasern, wodurch die Axenfibrillen weniger deutlich sichtbar werden. Nach Osmium-Behandlung zeigt nämlich die Grundsubstanz der Fasern ein dem in den Capillaren geronnenep Serum ähnliches optisches Verhalten, weshalb ich eben eine Verflussigung des Markes annehmen möchte. Dass dieses Entwickelungsstadium auch einer specifischen Veränderung des Protoplasmas entspricht, ersieht man aus dem Verhalten $z \mathfrak{u}$ den verschiedenen Farbstoffen. Einerseits hat es die Tinctionsfähigkeit der Plasmazelle für Anilin eingebuisst, anderseits das eigenthumliche Verhalten gegen Osmiumsäure noch nicht erlangt. Bei einem Theile dieser Fasern jedoch erscheint jene Verflussigung sehr wenig ausgesprochen, und die Axenfibrillen bleiben bis zum Auftreten des Markes deutlich sichtbar bestehen.

Die Bildung des Markes beginnt bereits vor der Umkleidung der jungen Axencylinder mit besonderen Scheiden, und tritt an den Fasern mit verflissigtem Inhalte in Form von Varicositäten, an den anderen von vornherein als mit dem Axencylinder parallel verlaufende Anlagerung auf (Osmiumpräparate). Häufig verkleben hiebei die sog. Remak'schen Quadratplättchen eines Axencylinders mit einer benachbarten noch marklosen Faser, woraus zu ersehen ist, dass die Markbildung durchaus nicht gleichzeitig an allen Axencylindern' einer Mutter-Faser auftritt. In dieser Weise entstandene Bündel junger Nervenfasern werden noch kurze Zeit von einer mehr oder weniger deutlich differenzirten Scheide um- 
schlossen ${ }^{1}$ ), welche als der nicht verflitssigte und nicht zum Mark umgewandelte Theil der Plasmazelle aufzufassèn ist.

Bei der Präparation lässt sich nicht selten eine solche junge Nervenfaser isoliren. Ist der Axencylinder noch nicht in seiner ganzen Länge vom Mark umgeben, so zeigt der marklose Theil mitunter eine pinselartige Auffaserung, welche orkennen lässt, dass der Axencylinder in diesem Entwickelungsstadium aus einer grossen Zahl von äusserst feinen Fibrillen zusammengesetzt ist.

Der Umstand, dass das Aussehen dieser, aus Plasmazellen entstandenen Stränge den sog. Remak'schen Fasern entspricht, veranlasste mich die Neubildung der Nerven im Froschsympathicus zu untersuchen. Hier zeigten mir schon die ersten Präparate die Anwesenheit von butndelweise angeordneten Axencylindern, welche sowohl in ihrer äusseren Erscheinung, als auch in ihrer Entwickelungsweise vollkommen mit der oben beschriebenen Form der Neubildung ubereinstimmten.

Dieser Uebergang der marklosen Fasern, welche den Nerven der wirbellosen Thiere zu vergleichen sind, in markhaltige, scheint mir auch für die Phylogenese von einem gewissen Interesse zu sein ${ }^{2}$.

Neben diesem Entstehungsmodus kommt im Ischiadicus des Frosches noch eine andere Art von Neubildung der Nervenfasern vor. Während der Nahrungsentziehung degeneriren viele Nervenfasern, indem sie allmählich ihr Mark verlieren, oder besser, das Mark wandelt sich in eine protoplasmatische Fltissigkeit um. Der Axencylinder schwindet ebenfalls, oder wird unsichtbar, und zwar in seiner ganzen Länge, oder nur auf einzelne Strecken, und erscheint dann in viele Stiicke zerfallen.

In der Folge, wenn der Frosch wieder in gute Ernährungsverhältnisse versetzt wird, entstehen auf eine mir nicht genau bekannte Weise, innerhalb der alten, diesen Process tiberlebt haben-

1) Diese bündelweise Anordnung der jungen Nerven hat bereits We issmann bei Embryonen beobachtet und in der Zeitschrift für rationelle Med. VII. Bd. S. 209 beschrieben.

2) Littré et Robin. Dictionn. de médicine. XI Edition. p. 1002. halten die Remak'schen Fasern für solche, díe auf einem früheren Entwicklungsstadium stehen geblieben sind, vgl. auch Sigmund Mayer: Die peripherische Nervenzelle und das sympathische Nervensystem. Arch. für Psychiatrie VI. Bd. 
Ueber die Entwickelung der Nerven aus Plasmazellen beim Frosche. 7

den Fasern, neue Nervenfasern, aus zwei oder aus mehreren Fäserchen zusammengesetzt. An diesen tritt das Mark ausschliesslich in der Form von exquisit varicöser Auflagerung auf. - Die letztere Art der Neubildung muss noch genauerem Studium unterworfen werden.

An den Gesammtscheiden der Nerven finden wir die Plasmazellen in den mannigfaltigsten Formen, runde oder mehr eckige, auf der inneren Fläche der Scheide schleierartig auflagernd; sie erreichen bisweilen sehr ansehnliche Dimensionen und wachsen nach allen Richtungen aus, indem sie nicht wie zwischen den Fasern in ihrer Wachsthumsrichtung gehindert werden. An den Riickenmarkshäuten kommen bisweilen ganze Geflechte von Plasmazellen vor, zwischen den Rickenmarksfasern und Zellen dagegen ist es mir nie gelungen solche Zellen aufzufinden.

Es bleibt mir schliesslich noch tubrig des Verfahrens zu gedenken, welches bei diesen Untersuchungen angewandt wurde. Wie vorhin erwähnt, erhielt ich die besten Präparate von Fröschen, die, nachdem sie ohne Nahrung 2 Monate (im Sommer) gehalten, in ein offenes geräumiges Springbrunnenreservoir versetzt, und ca. 4 Wochen gut ernährt worden waren; auf diese Weise gelang es mir die Plasmazellen förmlich zu ztlchten.

Die Nerven wurden zuerst mit Osmiumsäure 1:200 behandelt, und darauf mit schwach angesäuerter Lösung von amoniakalischem Carmin gefärbt. Die schönsten, leider vergänglichen Bilder liefert Fuchsin; in Glycerin zerzupft, muss das Object sofort unter das Microskop gebracht werden. Ein fitr die Aufbewahrung entschieden bestes Verfahren gibt Ehrlich an, nämlich die Färbung mit Dahlia, Methylviolet, Fuchsin und anderen Anilinfarben, mit nachherigem Entwässern in Alcohol und dem Einschlusse in Terpentin. Die Isolation im verharztem Terpentin geht so zu sagen von selbst vor sich, weil die Fasern in ihrer ganzen Länge dem Terpentin anhaften, in Folge dessen findet auch selten Zerreissung statt. Will man jedoch den Elementen ihre ursprüngliche Gestalt erhalten, so muss man die Gewebe zuerst in Osmium härten. Diese Modification empfehle ich um so mehr, als sie auch die Prtufungstinction auf Plasmazellen (Ehrlich) nicht beeinträchtigt. 
8 Dr. Korybutt-Daszkiewicz: Ueber die Entwickelung der Nerven etc.

\title{
Erklärung der Abbildungen auf Taf. I.
}

Fig. 1 u. 2. Plasmazellen aus dem Ischiadicus vom Frosch.

Fig. 3. Theilung des Kernes einer Plasmazelle.

Fig. 4. Stellung der Plasmazelle neben einem Kerne der Schwann'schen Scheide einer Nervenfaser, ein Verhältniss, welches sich öfters wiederholt.

Fig. 5. Auseinanderrücken der Kerne nach vorangegangener Theilung derselben.

Fig. 6. Plasmazelle zu einem Strange mit mehreren Kernen ausgewachsen; a Theilung des Kernes.

Fig. 7. Lagerungs-Verhältniss der Plasmazelle zu der Nervenfaser.

Fig. 8 u. 9. Aus Plasmazellen hervorgegangene protoplasmatische Stränge mit Kernen. Beginn der Längsstreifung. Fig. 9a Kern an der Seite der Faser, b eine durch Präparation hervorgerufene Spaltung.

Fig. 10. Protoplasmatischer Strang mit deutlicher Längsstreifung (Axenfbrillenbildung).

Fig. 11. Bildung von Nervenbündeln, a Axenfibrillen. b Fertige markhaltige Nervenfasern.

Fig. 12 u. 13. Aus einem Bündel isolirte Nervenfasern. 13. Der marklose Theil zeigt pinselartige Auffaserung (Axenfibrillen).

Fig. 14. a b c d Plasmazellen von der Innenseite der Gesammt-Nervenscheiden.

\section{Untersuchungen an Schmetterlingsrüsseln.}

\author{
Von \\ Wilhelm Breitenbach.
}

Hierzu Tafel II.

Vor einiger Zeit veröffentlichte ich im „Archiv für mikroskopische Anatomie", Band XIV, eine ,vorläufige Mittheilung tiber einige neue Untersuchungen an Schmetterlingsriusseln". Den Ausgangspunkt meiner Untersuchungen bildete der eigenthtimliche Bau 


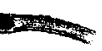
s.

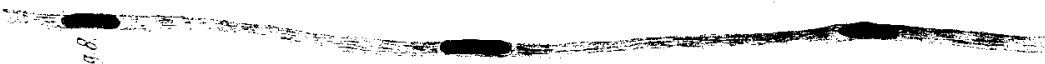
7.m.

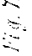

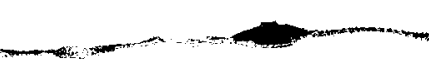
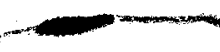

$\checkmark$

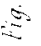

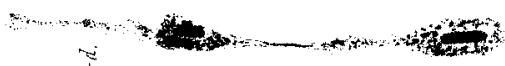

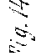

$\sum_{i}$

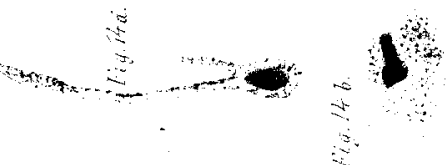

A.
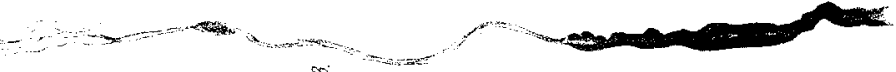

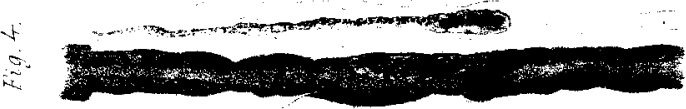

$\infty$
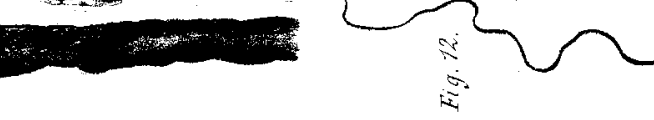

S.

$\therefore$ i

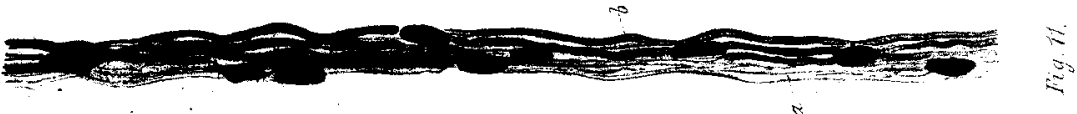

b

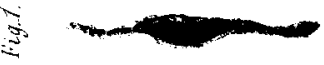

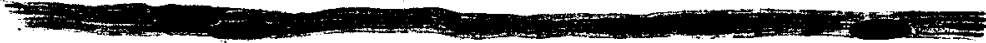

